\title{
URINARY N TELOPEPTIDE- THE IDEAL BIOMARKER FOR OSTEOPOROSIS
}

\author{
Ganesan G. Ram¹, N. Jambu ${ }^{2}$
}

${ }_{1}^{1}$ Associate Professor, Department of Orthopaedics, Sri Ramachandra Institute of Higher Education and Research, Chennai, Tamilnadu India.

2Professor, Department of Orthopaedics, Sri Ramachandra Institute of Higher Education and Research, Chennai, Tamilnadu India.

\section{BACKGROUND}

ABSTRACT

Osteoporosis and its complications cause a huge burden on that individual and the economy of the country. Hence diagnosing and treating osteoporosis is of paramount importance. At present, there are plenty of bone turnover markers available for monitoring the treatment and diagnosing osteoporosis. The aim of this study is to evaluate as to whether urinary $\mathrm{n}$ telopeptide is an ideal biomarker for diagnosing osteoporosis.

\section{METHODS}

This prospective case control study was done in Sri Ramachandra Institute of Higher Education and Research Hospital, Chennai, between August 2015 and August 2018. The study was approved by the institutional ethics committee. The study was done among volunteers who visited the hospital either as an inpatient or outpatient with suspected osteoporosis. The inclusion criteria werefemale more than 65 years and males more than 70 years and female less than 60 years and male less than 65 years having one or more risk factors for osteoporosis. All patients who participated in the study underwent both urinary $n$ telopeptide urine test and DEXA scan. Those patients whose DEXA report came as osteoporosis/osteopenia were taken as cases and those whose DEXA came as normal were considered as controls. We had sixty people as cases and fifty as controls. The collected data were analysed with IBM. SPSS statistics software 23.0 Version. To find the significant difference between the bivariate samples in independent groups, the unpaired sample t-test was used.

\section{RESULTS}

The mean urinary $\mathrm{n}$ telopeptide value for cases was 182.5 and control was 49.8 . The standard deviation for case and control were 159.9 and 31.3 respectively with standard error mean of 20.6 and 4.4 respectively. The mean urinary $n$ telopeptide values in the group 60 years and 65 years were statistically insignificant proving that urinary $n$ telopeptide test won't changes much once the patient attained menopause. The mean urinary n telopeptide values between male and female were statistically insignificant in both cases and control indicating that urinary $n$ telopeptide value won't change between gender and it mainly depends on bone resorption only.

\section{CONCLUSIONS}

Since urinary $\mathrm{N}$ telopeptide has less bio analytical non-modifiable variability, urinary $\mathrm{N}$ telopeptide can be considered as an ideal bone resorption biomarker.

\section{KEY WORDS}

Bone Biomarker, Urinary Marker, Fracture, DEXA Scan, Bone Turnover

HOW TO CITE THIS ARTICLE: Ram GG, Jambu N. Urinary N telopeptide- the ideal biomarker for osteoporosis. J. Evolution Med. Dent. Sci. 2019;8(26):2033-2036, DOI: 10.14260/jemds/2019/448

\section{BACKGROUND}

Osteoporosis is a silent killer causing a fracture every three seconds across the world. Osteoporosis and its complication cause a huge burden not only for that individual but also to the economy of the country. In European countries the burden and disability caused by osteoporosis are much worsen that that of cancer.(1) Osteoporosis not only affects women but almost equal number of men.(2) Hence diagnosing and treating Osteoporosis is a paramount importance. There should be a simple and cheap screening test or a diagnostic test for osteoporosis.

'Financial or Other Competing Interest': None.

Submission 21-03-2019, Peer Review 14-06-2019,

Acceptance 21-06-2019, Published 01-07-2019.

Corresponding Author:

Dr. Ganesan G. Ram,

Department of Orthopaedics,

No. 1, Ramachandra Nagar,

Porur, Chennai-600116, Tamilnadu, India.

E-mail: ganesangram@yahoo.com

DOI: $10.14260 /$ jemds $/ 2019 / 448$
At present the DEXA scan is the gold standard test available for diagnosing osteoporosis.(3) An ideal test should be economical, easily available, should be easily applicable to a community and should have good sensitivity and specificity. Even though DEXA scan has various advantages there are some disadvantages associated with it. Standardization of the reporting of DEXA scan is the main disadvantage. The factors that can give a false result are anatomy, artefacts, location of the machinery, machinery itself and it may also vary from technician to technician.(4) Scientific fraternity around the world is looking for a better test to replace the DEXA. At present there are plenty of Bone turnover markers available for monitoring the treatment and diagnosing osteoporosis.(5) Urinary $\mathrm{N}$ telopeptide is the stable degradation end product, which could be measured both in the serum and urine.(6,7) The urinary excretion is not affected by diet, and therefore shows less variation than the conventional markers. The NTx is specific to bone due to unique amino acid sequence and orientation of cross-linked alpha- $2 \mathrm{~N}$ Telopeptide. The aim of this study is to assess whether urinary $n$ telopeptide is an ideal biomarker for diagnosing osteoporosis. 


\begin{tabular}{|c|c|c|c|c|c|}
\hline NTx Value & N & Mean & Std. Dev & Std. Error Mean & p Value \\
\hline Cases & 60 & 182.540 & 159.9683 & 20.6 & \multirow{2}{*}{.0005} \\
\hline Controls & 50 & 49.839 & 31.3343 & 4.4 & \\
\hline \multicolumn{7}{|c|}{ Table 1. Mean NTx Value } \\
\hline
\end{tabular}

\begin{tabular}{|c|c|c|c|c|c|}
\hline Age & N & Mean & Std. Deviation & Std. Error Mean & p Value \\
\hline$<=60$ years & 17 & 169.59 & 103.193 & 25.028 & \multirow{2}{*}{.359} \\
\hline$>60$ years & 43 & 187.70 & 178.266 & 27.185 & \\
\hline \multicolumn{7}{|c|}{ Table } \\
\hline
\end{tabular}

\begin{tabular}{|c|c|c|c|c|c|}
\hline Age & N & Mean & Std. Deviation & Std. Error Mean & p Value \\
\hline$<=60$ years & 15 & 38.53 & 18.871 & 4.873 & \multirow{2}{*}{.294} \\
\hline$>$ 60 years & 35 & 54.69 & 34.459 & 5.825 & \\
\hline \\
Table 3. Mean N Telopeptide Value in Control at 60 Years \\
\hline
\end{tabular}

\begin{tabular}{|c|c|c|c|c|c|}
\hline Age & N & Mean & Std. Deviation & Std. Error Mean & p Value \\
\hline$<=65$ years & 30 & 156.77 & 99.397 & 18.147 & \multirow{2}{*}{.060} \\
\hline$>65$ years & 30 & 208.37 & 201.941 & 36.869 & \\
\hline \multicolumn{2}{|c|}{ Table 4. Mean N Telopeptide Value in Case at 65 Years } \\
\hline
\end{tabular}

\begin{tabular}{|c|c|c|c|c|c|}
\hline Age & N & Mean & Std. Deviation & Std. Error Mean & p Value \\
\hline$<=65$ years & 23 & 43.87 & 20.543 & 4.284 & \multirow{2}{*}{.107} \\
\hline$>65$ years & 27 & 54.93 & 37.873 & 7.289 & \\
\hline \multicolumn{7}{|c|}{ Table 5. Mean N Telopeptide Value in Control at 65 Years } \\
\hline
\end{tabular}

\begin{tabular}{|c|c|c|c|c|c|}
\hline Sex & $\mathbf{N}$ & Mean & Std. Dev & Std. Error Mean & p Value \\
\hline M & 17 & 210.12 & 229.700 & 55.710 & \multirow{2}{*}{.032} \\
\hline F & 43 & 171.67 & 124.104 & 18.926 & \\
\hline \multicolumn{7}{|c|}{ Table 6. Mean NTx Value- Male and Female in Case } \\
\hline
\end{tabular}

\begin{tabular}{|c|c|c|c|c|c|}
\hline Sex & N & Mean & Std. Dev & Std. Error Mean & p Value \\
\hline M & 23 & 56.22 & 40.105 & 8.363 & \multirow{2}{*}{.039} \\
\hline F & 27 & 44.41 & 20.549 & 3.955 & \\
\hline \multicolumn{7}{|c|}{ Table 7. Mean NTx Value-Male and Female in Control } \\
\hline
\end{tabular}

\section{METHODS}

Prospective case control study was done in Sri Ramachandra Institute of higher education and research Hospital, Chennai between August 2015 to August 2018. The study was approved by institutional ethics committee. The study was done among the volunteers who visited the Hospital either as an inpatient or outpatient. The inclusion criteria were female more than 65 years and males more than 70 years and female less than 60 years and male less than 65 years having one or more risk factors for osteoporosis. Patients of that particular age group were included as only these age group falls under pre requisite for doing DEXA scan for osteoporosis screening as per NICE guidelines.(8) The risk factors were patients having body mass index less than twenty, Asian, sedentary life style, osteoporosis or fragility fracture history in family members, sustained previous fracture or a recent fragility fracture, prolonged steroid use, more than two to three alcohol drinks per day, early menopause below forty-five years, vitamin D deficiency, depletion of testosterone hormones in male and neurological illness like dementia which makes person prone to fall. The exclusion criteria were persons having pathological fractures, hyperparathyroidism, hyperthyroidism and disease affecting bone metabolism like hepatic and renal failures and on drugs affecting bone metabolism. The sample size was calculated using single proportion relative precision technique and was calculated as 43. All the patients who participated in the study underwent both urinary $\mathrm{n}$ telopeptide urine test and DEXA scan. Those patients whose DEXA report came as osteoporosis/osteopenia were taken as case and those whose DEXA came as normal were considered as control. We had sixty people as cases and fifty as control.
DEXA scan was done using GE prodigy pro DEXA machine. DEXA scan was done in Right hip for all patients. If patient was having a fracture in the right hip or any surgeries done on right hip then DEXA scan was done in left hip.(9,10) The most vital biologic elements that can influence the bone and urinary bone markers were day and night variability and everyday changeability in bone metabolism. Amount of bone turnover markers were most elevated in the early morning and least toward the evening and night. Hence for all our patients, blood was taken in early morning. Urinary sample was collected for 24 hours from 6 am to next day 6 am in a sterile plastic container. Urine sample was analysed by Elisa technique for urinary $\mathrm{n}$ telopeptide level.(11) Biochemical test were done at Centralized Sri Ramachandra Hospital laboratory in our medical centre. The results from DEXA Scan were taken as gold standard against urinary $\mathrm{n}$ telopeptide and statistical evaluation were done.

\section{Statistical Analysis}

The collected data were analysed with IBM.SPSS statistics software 23.0 Version. To find the significant difference between the bivariate samples in Independent groups the Unpaired sample t-test was used. In both the above statistical tools the probability value 0.05 is considered as significant level.

\section{RESULTS}

We had 110 people participated in this study. Taking DEXA scan report as gold standard sixty people who had either Osteopenia or Osteoporosis forms the case group and remaining 50 people who had DEXA report normal formed the control group. We had forty-three female and seventeen males in the case group and twenty-seven female and twentythree male in the control group. We had thirty people in the case group and twenty-seven people in the control group who were more than sixty-five years while remaining were between fifty-five to sixty-five. The mean urinary $n$ telopeptide value for cases was 182.5 and control was 49.8 as tabulated in table 1 . From table 1 it is evident that the independent sample test for urinary $\mathrm{n}$ telopeptide values between Cases and control were statistically significant.

The tables 2,3,4,5 exemplify the mean values of Urinary $\mathrm{N}$ telopeptide in various age groups in the cases and the control group. The mean values of urinary $\mathrm{N}$ telopeptide in the age group above and below sixty were 187.70 and 169.59 respectively. Similarly, the mean urinary $\mathrm{N}$ telopeptide values in the age group above and below sixty-five were 208.37 and 156.77 respectively.

The table 6 and 7 encapsulates the mean value of urinary $\mathrm{N}$ telopeptide between male and female in the case and control group. The mean urinary $\mathrm{n}$ telopeptide values between male and female were statistically insignificant from tables 6.

\section{DISCUSSION}

Least bone mass and the micro-architectural degeneration of the bone tissue are the two main features of osteoporosis indicating the high risk of fracture. In the diagnosis of osteoporosis, the bone mineral density determined by the Dual x-ray absorptiometry is the best and a reliable mode of diagnosis, at the same time the calculations of the BMDs do not measure all the risk factors of fracture. The serum 
measurement and the urinary biochemical bone turnover markers are used to assess the skeletal turnover in a noninvasive and a much easier way. Bone biomarkers are delivered from the bone renovating process included bone formation biomarkers, bone resorption biomarkers and bone turnover regulators.(12,13) Whenever there is a bone remodelling the high bone turnover accelerates the bone loss and thereby increasing the fracture risk in patients. These high turnovers are frequently evaluated by bone markers. Bone turnover rate and the properties of bone matrix proteins can be assessed at every clinical site by the measurement of bone metabolic markers and bone matrix markers in serum and urine.(14) Now a day's earmarked Biochemical Immunoassays markers were of adequately explicit and advantageous for clinical use.(15) There were various urinary and blood biochemical markers available for diagnosing, treatment and screening of osteoporosis and its main complication fractures.

The main disadvantages of these bone turnover markers are its preanalytical variability. The clinical interpretation mainly depends on it. These variabilities were broadly divided into non-modifiable and modifiable factors. Some of the non-modifiable factors are age, sex while the modifiable factors are exercise, circadian etc. A good biochemical bone marker should have minimal non-modifiable bio variability.(16,17) Even though if you take extreme steps to control that factor there will be some amount of variability that cannot be neutralised. Out of all bone turnover markers urinary $\mathrm{n}$ telopeptide had ideal characters making it as one of the important markers that can predict fracture. Biochemical markers are significantly higher in children than adults, particularly in the first year of life and at puberty when they increase to levels two to ten times the levels found in adults. After mid-puberty levels decrease toward adult levels; however, they probably do not reach a nadir until the fourth decade or later. In men the majority of markers do not change with age in subsequent years. (18) In women there is a marked increase in markers of bone turnover at the menopause.(19) Urinary $n$ Telopeptide range is almost fixed in the particular range once patient attains menopause unless there is bone resorption activity. The study participants in our study group were postmenopausal patients only and there was no age bias while measuring the mean value of urinary $n$ telopeptide.

On comparison the biochemical markers are found higher in children than in the adults especially the levels are raised from two to tenfold times in the first year of life and at puberty and slowly reduces in the mid-puberty and do not reach its normal till the fourth decade. Also, it was found in comparison that the markers show no variation in men irrespective of their age but in women there is a notable increase in bone turnover markers at the menopausal period. The statistical analysis was done by independent sample test for the mean urinary $n$ telopeptide values in the both groups 60 and 65 years and were found to be statistically insignificant proving that urinary $\mathrm{n}$ telopeptide test won't changes much once the patient attained menopause. The premenopausal women and a normal male have the similar values of urinary NTx/ Creatinine. $(20,21)$ The bone resorption is the main culprit for decrease in bone mass once the female attains her menopause. Menopause causes a depletion of oestrogen which in turn increases the Urinary NTx/creatinine values. The bone mass in men can also decreases once the age increases.(22) It has been suggested that a reduction in bone formation may be the hallmark of bone loss in men.(23) Markers levels tend to be higher in young men in the third and fourth decades than in young women, but in older men the levels tend to be lower than in postmenopausal women. There was a general assumption that the both urinary and blood biomarkers of resorption varies between male and female. The mean urinary $n$ telopeptide values between male and female were analysed using independent sample test for gender and found to be statistically insignificant in both cases and control indicating that urinary $\mathrm{n}$ telopeptide value won't change between gender and it mainly depends on bone resorption only. The limitations of this study were small sample size, single centre study and involve only south Indian patients.

\section{CONCLUSIONS}

Since urinary $\mathrm{N}$ telopeptide has less bio analytical variability and non-modifiable factors, urinary $\mathrm{N}$ telopeptide can be considered as an ideal bone resorption biomarker.

\section{REFERENCES}

[1] Johnell 0, Kanis JA. An estimate of the worldwide prevalence and disability associated with osteoporotic fractures. Osteoporosis International 2006;17(12):1726-33.

[2] Center JR, Nguyen TV, Schneider D, et al. Mortality after all major types of osteoporotic fracture in men and women: an observational study. The Lancet 1999;353(9156):878-82.

[3] Pisani P, Renna MD, Conversano F, et al. Screening and early diagnosis of osteoporosis through X-ray and ultrasound based techniques. World Journal of Radiology 2013;5(11):398-410.

[4] Carlson-Newberry SJ, Costello RB. Emerging technologies for nutrition research: potential for assessing military performance capability. Institute of Medicine (US) Committee on Military Nutrition Research. Washington (DC): National Academies Press (US) 1997. ISBN-10: 0-309-05797-3.

[5] Shetty S, Kapoor N, Bondu JD, et al. Bone turnover markers: emerging tool in the management of osteoporosis. Indian Journal of Endocrinology and Metabolism 2016;20(6):846-52.

[6] Hanson DA, Weis MA, Bollen AM, et al. A specific immunoassay for monitoring human bone resorption: Quantitation of type I collagen cross-linked $\mathrm{N}$-telopeptides in urine. Journal of Bone and Mineral Research 1992;7(11):1251-8.

[7] Bonde M, Qvist P, Fledelius C, et al. Immunoassay for quantifying type 1 collagen degradation products in urine evaluated. Clinical Chemistry 1994;40(11 Pt 1):2022-5.

[8] Rabar S, Lau R, O’Flynn N, et al. Risk assessment of fragility fractures: summary of NICE guidance. BMJ 2012;345:e3698.

[9] Kanis JA, Oden A, Johnell O, et al. The burden of osteoporotic fractures: a method for setting intervention thresholds. Osteoporosis International 2001;12(5):417-27.

[10] Kanis JA. Diagnosis of osteoporosis and assessment of fracture risk. The Lancet 2002;359(9321):1929-36. 
[11] Gubala V, Harris LF, Ricco AJ, et al. Point of care diagnostics: status and future. Analytical Chemistry 2012;84(2):487-515.

[12] Liu L, Webster TJ. In situ sensor advancements for osteoporosis prevention, diagnosis and treatment. Current Osteoporosis Reports 2016;14(6):386-95.

[13] Kuo TR, Chen CH. Bone biomarker for the clinical assessment of osteoporosis: recent developments and future perspectives. Biomarker Research 2017;5(1):18.

[14] Delmas PD, Eastell R, Garnero P, et al. The use of biochemical markers of bone turnover in osteoporosis. Osteoporosis International 2000;11(Suppl 6):S2-S17.

[15] Kleerekoper M, Edelson GW. Biochemical studies in the evaluation and management of osteoporosis: current status and future prospects. Endocrine Practice 1996;2(1):13-9.

[16] Lombardi G, Lanteri P, Colombini A, et al. Blood biochemical markers of bone turnover: pre-analytical and technical aspects of sample collection and handling. Clinical Chemistry and Laboratory Medicine 2012;50(5):771-89.

[17] Greenblatt MB, Tsai JN, Wein MN. Bone turnover markers in the diagnosis and monitoring of metabolic bone disease. Clinical Chemistry 2017;63(2):464-74.
[18] Fatayerji D, Eastell R. Age-related changes in bone turnover in men. Journal of Bone and Mineral Research 1999;14(7):1203-10.

[19] Khosla S, Atkinson EJ, Melton LJ 3rd, et al. Effects of age and estrogen status on serum parathyroid hormone levels and biochemical markers of bone turnover in women: a population-based study. The Journal of Clinical Endocrinology \& Metabolism 1997;82(5):1522-7.

[20] Ebeling PR, Atley LM, Guthrie JR, et al. Bone turnover markers and bone density across the menopausal transition. The Journal of Clinical Endocrinology \& Metabolism 1996;81(9):3366-71.

[21] Bell NH, Hollis BW, Shary JR, et al. Diclofenac sodium inhibits bone resorption in postmenopausal women. The American Journal of Medicine 1994;96(4):349-53.

[22] Jones G, Nguyen T, Sambrook P, et al. Progressive loss of bone in the femoral neck in elderly people: longitudinal findings from the Dubbo osteoporosis epidemiology study. BMJ 1994;309(6956):691-5.

[23] Wishart JM, Need AG, Horowitz M, et al. Effect of age on bone density and bone turnover in men. Clinical Endocrinology 1995;42(2):141-6. 